\title{
关于调和同态的某些注记 *
}

\author{
东瑜昕 \\ (杭州大学数学系, 杭州 310028)
}

\author{
程杞元 \\ (北京理工大学应用数学系, 北京 100081)
}

\section{关键词调和同态 临界点 符号}

Riemann 流形之间将调和函数芽拉回到调和函数芽的映射称为调和同态, 它等价于水平 弱共形调和映射 ${ }^{[1,2]}$. 特殊流形之间调和同态的分类、构造是主要问题, 已有很多调和同态的 有趣的例子(参见文献 $[3 \sim 7]$ 和 Gudmundsson 的文章 ${ }^{1)}$ ).

研究调和同态的整体性质必涉及临界点集的性质. 本文首先利用调和同态的符号 (symbol) 研究了流形维数对临界点集的影响, 然后考察了 $\varphi: M^{n+1} \rightarrow N^{n}$ 情形的调和同态. 当 $n=$ 2 时 Baird 和 Wood ${ }^{[3]}$ 建立了完美的理论. 但是当 $n \geqslant 3$ 时, 我们说明 $\varphi$ 的存在性往往遇到拓 扑障碍. 我们给出一些整体的不存在性定理.

\section{1 准备知识}

设 $\varphi:\left(M^{m}, g\right) \rightarrow\left(N^{n}, h\right)$ 是 $\mathrm{C}^{\infty}$ 映射. 令 $C_{\varphi}:=\left\{x \in M \mid \mathrm{d} \varphi_{x}=0\right\}, M^{*}:=M \backslash C_{\varphi}$. 在每一点 $p \in M^{*}$, 我们有垂直空间 $V_{p}:=\operatorname{Ker} \mathrm{d} \varphi_{p} \subset T_{p} M$ 和水平空间 $H_{p}:=V_{p}^{\perp}$. 如果对于 任意 $p \in M^{*},\left.\mathrm{~d} \varphi\right|_{H p}: H_{p} \rightarrow T_{\varphi(p)} N$ 是满的共形映射, 则称 $\varphi$ 为水平弱共形的.

引理 $\mathbf{A}^{[1,2]}$ 映射 $\varphi: M^{m} \rightarrow N^{n}$ 是调和同态当且仅当 $\varphi$ 是水平弱共形调和映射.

因此, 如果 $\varphi$ 是非常值的调和同态必有 $m \geqslant n$. 从文献[1]知道一个非常值调和同态 $\varphi$ 的 临界点集 $C_{\varphi}$ 是 $M$ 中的极集. 极集的一个性质是它不能把 $M$ 中任意的开球邻域划分为不连 通. 特别地, 如果 $M$ 和 $N$ 是实解析时, $C_{\varphi}$ 是余维数不小于 2 的实解析集. 当目标流形是 2 维 曲面时, 我们有下列重要性质:

引理 $\mathbf{B}^{[8]}$ 设 $\varphi: M^{n} \rightarrow N^{2}$ 是水平共形浸没, 则 $\varphi$ 是调和的, 当且仅当 $\varphi$ 的纤维 $\varphi^{-1}(y)$ $\left(y \in N^{2}\right)$ 是 $M$ 中的极小子流形.

现设 $\varphi: M^{m} \rightarrow N^{n}$ 是非常值调和同态, $x_{0} \in C_{\varphi}$. 设 $U, V$ 分别是 $x_{0}$ 和 $\varphi\left(x_{0}\right)$ 的测地凸 邻域. 利用指数映照 $\exp _{x_{0}}: T_{x_{0}} M \rightarrow M$ 和 $\exp _{\varphi\left(x_{0}\right)}: T_{\varphi\left(x_{0}\right)} N \rightarrow N$, 我们可以建立分别以 $x_{0}$ 和 $\varphi\left(x_{0}\right)$ 为中心的法坐标系 $\left(U ; x^{1}, \cdots, x^{m}\right)$ 和 $\left(V ; y^{1}, \cdots, y^{n}\right)$. 记 $\varphi^{k}=y^{k} \circ \varphi, k=1, \cdots, n$;

1995-10-20 收稿, 1996-04-19 收修改稿

* 国家自然科学基金和浙江省自然科学基金资助项目

1) Gudmundsson S. The geometry of harmonic morphisms. Ph D Thesis, University of Leeds, 1992 
$\mathrm{D}_{\alpha}:=\left(\frac{\partial}{\partial x^{1}}\right)^{\alpha_{1}} \cdots\left(\frac{\partial}{\partial x^{m}}\right)^{\alpha_{m}}$. 令

$O_{x_{0}}(\varphi)=\min \left\{q\right.$; 对某个 $k$ 有 $\left.\mathrm{D}_{\alpha} \varphi^{k}(0) \neq 0,|\alpha|=\alpha_{1}+\cdots+\alpha_{m}=q\right\}$.

我们称 $O_{x_{0}}(\varphi)$ 为 $\varphi$ 在 $x_{0}$ 的阶数.

设 $\varphi$ 在 $x_{0}$ 的阶数为 $p$, 则可以定义 $\varphi$ 在 $x_{0}$ 的符号如下 ${ }^{[1]}$ :

$$
\xi=\left(\xi^{1}, \cdots, \xi^{n}\right): T_{x_{0}} M \rightarrow T_{f\left(x_{0}\right)} N \text {, }
$$

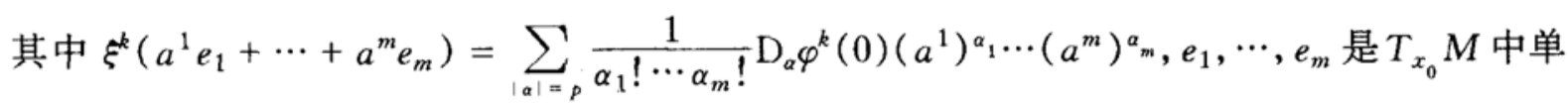
位正交基. 调和同态 $\varphi$ 与其符号 $\xi$ 的关系就是 Taylor 展开 ${ }^{[1]}$ :

$$
\begin{array}{ll}
\varphi^{k}(x)=\xi^{k}(x)+o\left(\gamma^{p+1}\right), & r=\sqrt{\left(x^{1}\right)^{2}+\cdots+\left(x^{m}\right)^{2}}, \\
\mathrm{D}_{i} \varphi^{k}(x)=\mathrm{D}_{i} \xi^{k}(x)+o\left(\gamma^{p}\right), & i=1, \cdots, m ; k=1, \cdots, n ; \mathrm{D}_{i}=\partial / \partial x^{i} .
\end{array}
$$

引理 $\mathrm{C}^{[1]} \xi: T_{x_{0}} M \rightarrow T_{\varphi\left(x_{0}\right)} N$ 是调和同态, 其分量为 $p$ 阶齐次多项式.

显然原点 $O$ 是 $\xi$ 的 $p$ 阶临界点. 如果 $\xi$ 有另外一个临界点 $\bar{x}$, 则由于 $\mathrm{D}_{i} \xi(i=1, \cdots, n)$ 是 $p-1$ 阶齐次多项式, 过 $O$ 与 $\bar{x}$ 的直线 $L$ 上点都是 $\xi$ 的临界点, 即 $L \subset C_{\xi}$. 因此, 如果 $O$ 是 $\xi$ 的孤立临界点,则 $C_{\xi}=\{O\}$. 此时, 我们称 $x_{0}$ 为 $\varphi$ 的广义退化临界点 ${ }^{[8]}$.

引理 1 如果 $x_{0}$ 是 $\varphi$ 的广义非退化临界点, 则 $x_{0}$ 必是孤立临界点.

证 如果 $x_{0}$ 不是孤立临界点, 则有点列 $\left\{x_{k}\right\}, x_{k} \in C_{\varphi}$ 使得 $x_{k} \rightarrow x_{0}$, 从(3)式可得

$$
O=\mathrm{D}_{i} \xi^{k}\left(x_{l}\right)+o\left(\gamma^{p}\right) \text {. }
$$

$\frac{x_{l}}{\left|x_{l}\right|}:=\tilde{x}_{l} \in S^{m-1},\left\{\tilde{x}_{l}\right\}$ 有收玫子列, 仍记作 $\tilde{x}_{l} \rightarrow \tilde{x} \in S^{m-1}$. 因为 $\mathrm{D}_{i} \xi^{k}$ 是 $p-1$ 阶齐次多 项式, 所以从 (4)式可得

$$
O=\gamma^{p-1} \mathrm{D}_{i} \xi^{k}\left(\tilde{x}_{l}\right)+o\left(\gamma^{p}\right)
$$

即

$$
o=\mathrm{D}_{i} \xi^{k}\left(\tilde{x}_{l}\right)+o(\gamma) .
$$

因此, $\mathrm{D}_{i} \xi^{k}\left(\widetilde{x}_{l}\right)=0, i=1, \cdots, m, k=1, \cdots, n$. 这与 $x_{0}$ 是广义非退化临界点矛盾.

\section{2 主要结果}

命题 1 设 $\varphi: M^{m} \rightarrow N^{n}$ 是非常值调和同态, $x_{0} \in C_{\varphi}$ 是 $p$ 阶临界点, $\xi(x)$ 是 $\varphi$ 在 $x_{0}$ 的 符号. 则 $O$ 是 $\xi(x)$ 的孤立零点当且仅当 $m-2=p(n-2)$. 此时, $x_{0}$ 必是 $\varphi$ 的广义非退化 临界点.

证 将 $T_{x_{0}} M$ 和 $T_{\varphi\left(x_{0}\right)} N$ 分别与 $\mathbb{R}^{m}$ 和 $\mathbb{R}^{n}$ 自然恒同, 则 $\xi: \mathbb{R}^{m} \rightarrow \mathbb{R}^{n}$ 是 $p$ 阶齐次多项式给出 的调和同态. 因为 $x_{0}$ 是临界点, 所以 $p \geqslant 2$.

用 $\lambda^{2}=\frac{|\mathrm{d} \xi|^{2}}{n}$ 表示 $\xi$ 的伸缩. 适当规范化以后不妨设 $\sup _{|\mathbf{x}|}|\xi(x)|=1$, 直接计算(参见 文献[9])可得

$$
\nabla S^{m-1}|\xi(x)|^{2}=2 \sum_{k=1}^{n} \xi^{k} \nabla \xi^{k}-2 p|\xi|^{2} x
$$




$$
\Delta s^{m-1}|\xi(x)|^{2}=2 n \lambda^{2}-2 p(2 p+m-2)|\xi|^{2} .
$$

对于任意临界点, Baird 证明了 $m-2 \geqslant p(n-2)$.

现设 $O$ 为 $\xi(x)$ 的孤立零点, 由齐性可知 $\left.|\xi(x)|^{2}\right|_{S^{m-1}} \quad>0, x \in S^{m-1}$. 连续函数 $|\xi(x)|^{2}$ 在 $S^{m-1}$ 上某点 $x_{1}$ 取到极小值. 因此,

$$
\nabla s^{m-1}\left|\xi\left(x_{1}\right)\right|^{2}=0, \Delta s^{m-1}\left|\xi\left(x_{1}\right)\right|^{2} \geqslant 0 .
$$

从(6), (7)式得

$$
\begin{gathered}
\sum_{k=1}^{n} \xi^{k} \nabla \xi^{k}=p|\xi|^{2} x_{1}, \\
n \lambda^{2} \geqslant p(2 p+m-2)|\xi|^{2} .
\end{gathered}
$$

根据文献 $[1],\left\langle\nabla \xi^{k}, \nabla \xi^{l}\right\rangle=\lambda^{2} \delta^{k l}$. 从(8) 式得 $\lambda^{2}=p^{2}|\xi|^{2}$ (在 $x_{1}$ 点), 代入(9) 式我们有 $m-2 \leqslant p(n-2)$. 因此, $m-2=p(n-2)$.

反过来, 如果 $m-2=p(n-2)$. 根据文献 [9], 我们知道 $\xi: S^{m-1} \rightarrow S^{n-1}$. 由 $\xi$ 的齐性 可见 $O$ 为 $\xi(x): \mathbb{R}^{m} \rightarrow \mathbb{R}^{n}$ 的孤立零点. 现再证 $O$ 为孤立临界点. 如果 $\xi$ 有临界点 $\bar{x} \neq 0$, 即 $\nabla \xi(\bar{x})=0$. 利用齐次函数的 Euler 公式

$$
\sum_{i=1}^{m} x^{i} \cdot \frac{\partial \xi}{\partial x^{i}}=p \xi(x),
$$

由于 $\xi(\bar{x})=0$, 这与 $O$ 为孤立零点矛盾.

推论 1 设 $x_{0}$ 是非常值调和同态 $\varphi: M^{m} \rightarrow N^{n}$ 的 $p$ 阶临界点. 如果 $x_{0}$ 不是 $\varphi$ 的广义非 退化临界点, 则必有 $m-2>p(n-2)$.

命题 2 设 $\varphi: M^{2(n-1)} \rightarrow N^{n}$ 是非常值调和同态, 则 $\varphi$ 最多只有广义非退化临界点.

证 因为 $\varphi$ 非常值, 故 $n \geqslant 2$. 若 $x_{0}$ 是 $\varphi$ 的 $p$ 阶临界点, 则从文献[10] 我们知道 $2(n-$ $1)-2 \geqslant p(n-2)$, 即 $p \leqslant 2$. 若 $p \leqslant 1$, 则 $x_{0}$ 不是临界点. 因此, $p=2$. 于是, 从命题 1 可 知 $x_{0}$ 是广义非退化临界点.

注 1 (a) 如果 $n=2, \varphi: M^{2} \rightarrow N^{2}$ 就是弱共形调和映射, 它只有孤立临界点是熟知的结 果. (b) $n=3$ 时, 我们就知 $\varphi: M^{4} \rightarrow N^{3}$ 的临界点是广义非退化的.

定理 1 设 $\varphi: M^{n+1} \rightarrow N^{n}$ 调和同态, 如果 $M$ 是下列情形之一:

(i) $M^{n+1}=S^{n+1}, n$ 为奇数且 $n \geqslant 4$;

(ii) $M^{n+1}=C P^{k}, k$ 为偶数且 $2 k-1 \geqslant 4$;

(iii) $M^{n+1}=H P^{l}, l$ 为偶数且 $4 l-1 \geqslant 4$, 这里 $S^{n+1}, C P^{k}, H P^{l}$ 上赋予任意 Riemann 度量, 则 $\varphi$ 必是常值.

证设 $\varphi: S^{n+1} \rightarrow N^{n}$ 是一调和同态. 如果 $\varphi$ 是非常值. 因为 $n \geqslant 4$, 从文献 [9] 中的纤维 化判定条件我们知道 $\varphi$ 没有临界点, 且确定了 $S^{n+1}$ 上一个光滑的、没有不动点的 $S^{1}$ - 作用. 这个 $S^{1}$ - 作用就是将 $S^{n+1}$ 上点沿着 $\varphi$ 的纤维移动. 记 $\mathrm{e}^{\mathrm{i} \theta} \in S^{1} ; f_{\theta}=\mathrm{e}^{\mathrm{i} \theta}: S^{n+1} \rightarrow S^{n+1}$ 表示此 $S^{1}$ - 作用. $f_{\theta}$ 是连续依赖于 $\theta$ 的一族映射, 因此 $\operatorname{deg} f_{\theta}=\operatorname{deg} f_{0}=1$. 由假设 $n$ 为奇数, $f_{\theta}$ 的 Lefschetz 数 $\Lambda_{f_{0}}=1+(-1)^{n+1}=2 \neq 0$. 根据 Lefschetz 不动点定理, $f_{\theta}$ 必有不动点, 与上述 矛盾. 这样就证明了 (i). 类似地, 可以证明(ii), (iii). 
注 $2 n$ 为偶数时, Hopf 纤维投影 $S^{n+1} \rightarrow C P^{n / 2}$ 是非常值调和同态.

定理 2 设 $M^{4}$ 是 Einstein 流形. 则任何非常值的调和同态 $\varphi: M^{4} \rightarrow S^{3}$ 决定了 $M^{4}$ 上一 个(可积的) Hermite 结构.

证 设 $\pi: S^{3} \rightarrow S^{2}$ 是 Hopf 投影, 它是 Riemann 浸没的调和同态. 令 $\psi=\pi \circ \varphi: M^{4} \rightarrow S^{2}$. 则 $\psi$ 仍是调和同态 ${ }^{[1]}$. 用 $J^{\mathrm{H}}, J^{\mathrm{V}}$ 分别表示在 $\psi$ 的水平空间和垂直空间中关于某个定向旋转 $+\pi / 2$. 则可以在 $M \backslash C_{\psi}$ 上定义两个近 Hermite 结构: $J^{1}=\left(J^{\mathrm{H}}, J^{\mathrm{v}}\right)$ 和 $J^{2}=\left(J^{\mathrm{H}},-J^{\mathrm{V}}\right)$. 当 $M^{4}$ 是 Hermition 时, Wood ${ }^{[7]}$ 证明了 $J^{1}$ 和 $J^{2}$ 中有一个是可积的. 且当 $C_{\psi}$ 中点都是广义非退化 的临界点时, 此可积的 Hermite 结构可以光滑地延拓到整个 $M^{4}$ 上. 由于 $\pi$ 是 Riemann 浸没, $C_{\psi}=C_{\varphi}$. 根据命题 $2, \psi$ 的临界点都是广义非退化的.

推论 2 如果 $M^{4}$ 是下列情形之一:

(i) $M^{4}=S^{4}$;

(ii) $M^{4}=C P^{2}$.

则任何调和同态 $\varphi: M^{4} \rightarrow S^{3}$ 必是常值.

证 (i) 因为 $S^{4}$ 上不存在 Hermite 结构. 根据定理 2 我们知道不存在非常值的调和同态 $\varphi: S^{4} \rightarrow S^{3}$.

(ii) 根据文献[7]中推论 $8.3, \pi \circ \varphi$ 为常值, 因此 $\varphi$ 为常值.

注 3 赋予 $S^{n+1}, S^{n}$ 任意 Riemann 度量, Baird ${ }^{[9]}$ 证明了任何调和同态 $\varphi: S^{n+1} \rightarrow S^{n}(n \geqslant$ 4) 是常值的. Baird 和 Ratto ${ }^{[10]}$ 证明在 $S^{4}$ 或者 $S^{3}$ 上赋予与标准度量共形的适当 Riemann 度 量时, 存在非常值调和同态 $\varphi: S^{4} \rightarrow S^{3}$.

\section{参考文 献}

1 Fuglede B. Harmonic morphisms between Riemannian manifolds. Ann Inst Fourier, 1978, 28: 107 144

2 Ishitara T. A mapping of Riemannian manifolds which preserves harmonic functions. J Math Kyoto Univ, 1979, 19: 215 229

3 Baird P, Wood J C. Harmonic morphisms, Serfert fibre spaces and conformal foliations. Proc London Math Soc, 1992,64 (3) : $170 \sim 196$

4 Baird P. Harmonic maps with symmetry, harmonic morphisms and deformation of metrics. Research Notes in Mathematics 87, London: Pitman, 1983

5 Eells J, Lemaire L. Selected topics in harmonic maps. CBMS Regional Conf Ser Math 50, Amer Math Soc, Providence, R I, 1983

6 Gudmundsson S, Wood J C. Multivalued harmonic morphisms. Math Scand, 1993, 73: 127 155

7 Wood J C. Harmonic morphisms and Hermitian structures on Einstein 4-manifolds. Inter J Math, 1992, 3: 415 439

8 Baird P. Eells J. A conservation law for harmonic maps, In: Geometry Symposium Utrecht 1980. Lecture Notes in Math 894, New York: Springer-Verlag, 1981. 1 25

9 Baird P. Harmonic morphisms and circle actions on 3- and 4-manifolds. Ann Inst Fourier, 1990, 40: 177 212

10 Baird P, Ratto R. Conservation laws, equivariant harmonic maps and harmonic morphisms. Proc London Math Soc, 1992, 64 (3): $197 \sim 224$ 